\title{
Correlates of perceived military to civilian transition challenges among Canadian Armed Forces Veterans
}

\author{
Jennifer E.C. Lee ${ }^{a}$, Sanela Dursun ${ }^{\mathrm{a}}$, Alla Skomorovsky a and James M. Thompson ${ }^{\mathrm{b}}$
}

\begin{abstract}
Introduction: Analyses of the Canadian Armed Forces Transition and Well-Being Survey (CAFTWS) were conducted to identify the most prominent challenges faced by Canadian Armed Forces (CAF) Veterans during their military to civilian transition, and to assess the importance of various characteristics, including release category and health status, as risk factors for experiencing such challenges. Methods: Prevalence estimates and logistic regression analyses were computed on data from the CAFTWS, which was administered in 2017 to 1,414 Regular Force Veterans released from the CAF in the previous year. Results: Two of seven perceived transition challenges had the strongest associations with difficult post-military adjustment: the loss of military identity (adjusted odds ratio $[A O R]=5.4$ ) and financial preparedness $(\mathrm{AOR}=2.3)$. In adjusted regression analyses, Veterans who had non-commissioned rank, who had primarily served in the army, had 10-19 years of service, had a medical release, and had poor physical or mental health, were more likely to report loss of military identity. Veterans who had junior non-commissioned rank, a medical release, and poor physical or mental health were more likely to report challenges with financial preparedness. Furthermore, we observed significant interaction effects between Veterans' release type and their health status. Discussion: This study extends prior research to inform ongoing efforts to support the well-being of CAF members adjusting to post-service life. Findings emphasize the importance of preparing transitioning service members and civilian communities for the social identity challenges they may encounter. Findings also support the value of programs and services that help prepare transitioning service members with managing finances, finding education and employment, relocating, finding health care providers, and understanding benefits and services.
\end{abstract}

Key words: Canadian Armed Forces, financial well-being, military identity, military to civilian transition, social identity, Veterans

\section{RÉSUMÉ}

Introduction: Les auteurs ont analysé l'Enquête sur la transition à la vie civile et le bien-être après le service dans les Forces armées canadiennes (ETBFAC) afin d'établir les principales difficultés qu’ont affrontées les vétérans des Forces armées canadiennes (FAC) lors de leur transition à la vie civile et d'évaluer l'importance de diverses caractéristiques, y compris le type de libération et l'état de santé, parmi les facteurs de risque de vivre ces difficultés. Méthodologie: Ils ont calculé les évaluations de prévalence et les analyses de régression logistique à partir des données de l'ETBFAC, à laquelle 1414 vétérans de la Force régulière libérés au cours de l'année précédente ont répondu en 2017. Résultats: Deux des sept difficultés perçues à la transition étaient étroitement liées à une adaptation éprouvante à la vie civile : la perte de l'identité militaire (rapport de cotes rajusté $=5,4$ ) et la préparation financière (rapport de cotes rajusté $=2,3$ ). Dans les analyses de régression rajustées, les vétérans qui avaient été sous-officiers, avaient surtout servi dans l'armée, avaient 10 à 19 ans de service, avaient été libérés pour des raisons médicales et étaient en mauvaise santé physique ou mentale étaient plus susceptibles de déclarer une perte d'identité militaire. Les vétérans qui étaient des sous-officiers subalternes, avaient été libérés pour des raisons médicales et étaient en mauvaise santé physique ou mentale étaient plus susceptibles de déclarer des difficultés relatives à leur préparation financière. De plus, on constatait des effets d'interaction importants entre le type de libération des vétérans et leur état de santé. Discussion: La présente étude élargit la portée de recherches antérieures pour éclairer les efforts en vue de soutenir le bien-être des membres des FAC qui s’adaptent à la vie après le service militaire. Les résultats font ressortir l'importance de préparer les militaires en transition et les groupes civils à d'éventuelles difficultés en matière d'identité sociale. Les résultats font également ressortir l'importance des programmes et des services qui contribuent à préparer les militaires en transition à gérer leurs finances, à trouver des

a Director General Military Personnel Research and Analysis, Department of National Defence, Ottawa, Ontario

b Department of Public Health Sciences, Queen's University, Kingston, Ontario

Correspondence should be addressed to Jennifer Lee at jennifer.lee@forces.gc.ca 
possibilités de formation et d'emploi, à déménager, à trouver un professionnel de la santé et à comprendre les avantages et les services.

Mots-clés : bien-être financier; Forces armées canadiennes; identité militaire; identité sociale; transition entre la vie militaire et la vie civile; vétérans

\section{INTRODUCTION}

Identified as an important event in the life course of military personnel, the transition from military to civilian life has received increasing attention in the literature. ${ }^{1-4}$ Indeed, the Well-being Framework adopted by the Canadian Armed Forces (CAF) and Veterans Affairs Canada (VAC) recognizes the impacts that this event can have across multiple domains of service members' well-being, including their health, employment or sense of purpose, finances, social integration, life skills and preparedness, housing and physical environment, and cultural and social environment. ${ }^{5,6}$ Since 2010, the Life After Service Studies (LASS) have been the leading source of information on the well-being of CAF Veterans during post-military adjustment. ${ }^{7-9}$ In the 2016 LASS, it was estimated that close to one-third of Veterans were experiencing, or had experienced, a difficult adjustment to civilian life, and the proportion was even greater among those who were released more recently. ${ }^{8}$

A 2012 report outlined some of the transition challenges that medically released CAF members face, including limited awareness about available support services and the high volume of paperwork. ${ }^{10}$ However, it is important to consider issues other than administrative challenges or type of release. While the LASS surveys have found that the majority of Veterans adjust well to civilian life, some important group variations have been noted. ${ }^{2,11,12}$ Analyses have also shown that post-military adjustment is strongly associated with mental health problems, regardless of the type of release; that a high proportion (60\%) of Veterans who report difficult postmilitary adjustment were not medically released; and that health and other well-being problems are prevalent among non-medically released Veterans who report difficult post-military adjustment. ${ }^{2,13}$ When assessing post-military adjustment, it is therefore important to consider the impacts on various domains of Veterans' well-being. Based on past studies, some of the challenges that Veterans may experience when adjusting to their post-service life include: loss of military identity, difficulty finding employment, problems related to living with chronic health conditions, and suicidal ideation. ${ }^{1,3,4,11,14-20}$ However, more detail about the population-wide extent and correlates of transition challenges is needed to inform the development of relevant support services and programs.

The Canadian Armed Forces Transition and WellBeing Survey (CAFTWS) was conducted to address gaps in knowledge about the challenges involved in adjusting to post-military life using a representative sample of newly released CAF Veterans. The objectives of this study were (1) to identify aspects of military to civilian transition that are perceived as challenging by CAF Veterans, and (2) to explore the associations of type of release and health status with commonly perceived transition challenges. The goal was to provide a clearer understanding of military to civilian transition challenges in order to inform planning for services supporting the well-being of CAF members at this important stage of life.

\section{METHODS}

\section{Sampling and data collection}

The CAFTWS was conducted by Statistics Canada between April and June 2017. It employed a stratified systematic random sampling of Veterans who transitioned out of the CAF in 2016 with a minimum of 2 years of service (730 days). The target sample was drawn from the CAF human resources database. Veterans who were released for misconduct or unsatisfactory service were excluded, owing to methodological challenges in accessing that group.

A total of 1,414 CAF Veterans participated in the CAFTWS, representing an estimated weighted population of 4,100 Veterans. They were all surveyed within 18 months of their release date via computer-assisted interviews (75\% response rate). Statistics Canada ensured the project met ethical guidelines, and participation required the provision of informed consent. More information on the survey sampling and data collection can be found elsewhere. ${ }^{21}$

\section{Survey instrument}

The CAFTWS survey instrument was developed by researchers within the Department of National Defence (DND) based on a pilot study ${ }^{22}$ in collaboration with Statistics Canada and VAC. 


\section{Perceived transition challenges}

Questions used to assess perceived transition challenges were adapted from a past survey. ${ }^{23}$ Participants indicated the extent to which they perceived 10 items as challenging during their transition (see Figure 1 for the list of items) using a 5-point Likert-type scale (1 = not at all challenging, 2 = a little challenging, 3 = moderately challenging, $4=$ very challenging, or $5=$ extremely challenging; not applicable was also provided as an option). Indicators were created to identify participants who perceived each item as challenging (i.e., they rated the item as having been either very challenging or extremely challenging during their transition).

\section{Difficult post-military adjustment}

Ease of post-military adjustment was assessed using a question drawn from the LASS. ${ }^{2}$ Participants were asked to rate their adjustment to civilian life since being released from the CAF on a 5-point Likert-type scale $(1=$ very easy, $2=$ moderately easy, 3 = neither easy nor difficult, $4=$ moderately difficult, $5=$ very difficult). An indicator was created to identify participants who

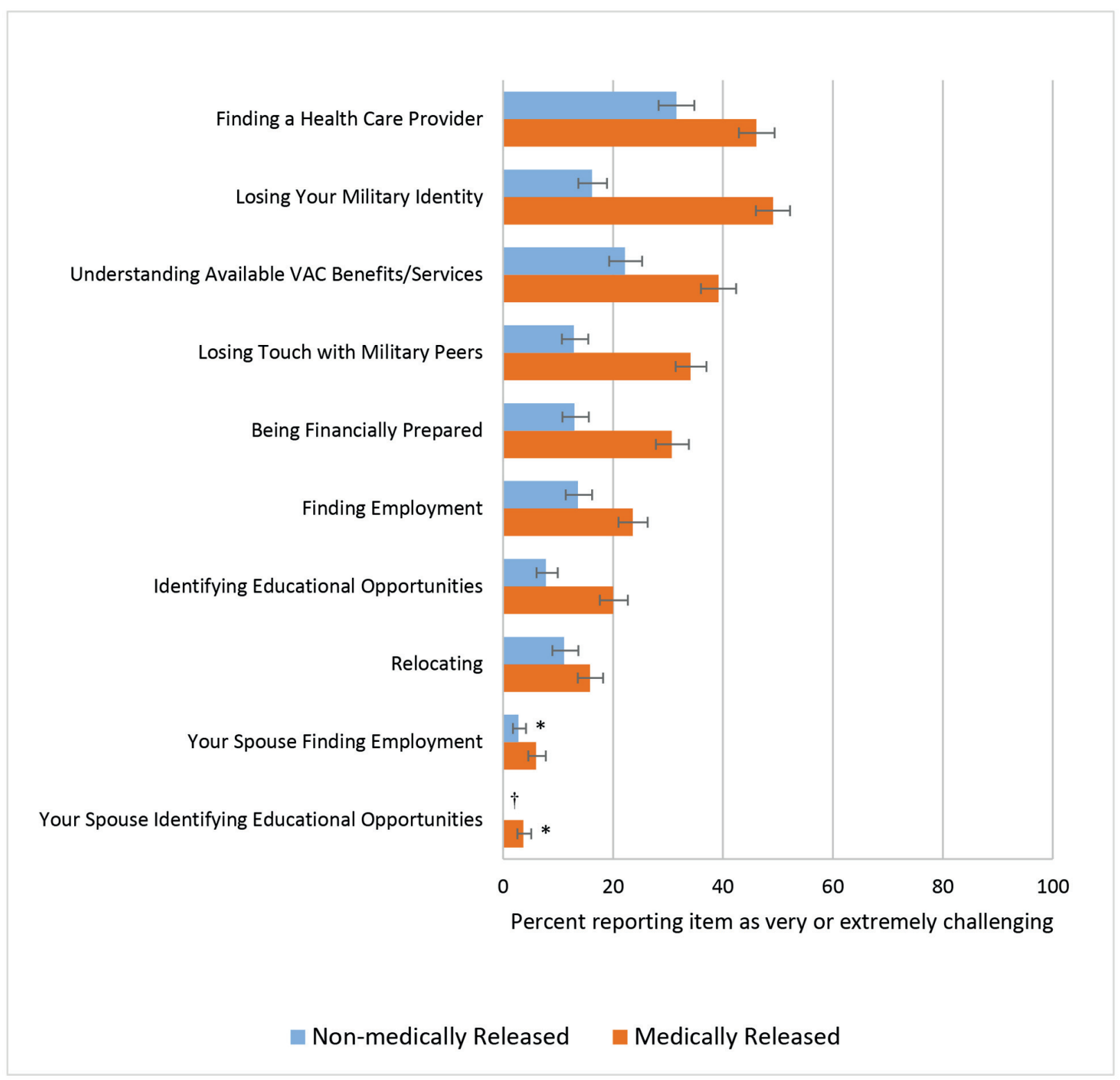

Figure 1. Perceived post-military transition challenges by release type

* Use with caution; coefficient of variation 16.6-33.3

† Too unreliable to report 
reported a difficult post-military adjustment (i.e., they rated their adjustment as moderately difficult or very difficult) from all other participants.

\section{Release type}

Participants were asked to identify the category under which they were released from the CAF (voluntary, medical, retirement age, involuntary, service complete, unknown, refusal). An indicator was created to distinguish participants who were medically released from all other participants.

\section{Self-rated health status}

In line with the approach taken in the Canadian Community Health Survey (CCHS), participants were asked to rate their physical and mental health on a 5-point Likert-type scale $(1=$ poor, $2=$ fair, $3=$ good, $4=$ very good, $5=$ excellent). They were then classified based on whether they rated their health poorly (poor or fair) or good (good, very good, or excellent).

\section{Demographic and military characteristics}

Demographic and military characteristics considered in the present study were age, sex, rank (junior noncommissioned member [NCM], senior NCM, junior officer, senior officer), element (Army, Navy, Air Force), Afghanistan deployment history (yes/no), history of other deployment outside of North America (yes/no), and years of service.

\section{Analyses}

Analyses were conducted using STATA statistical software (StataCorp, College Station, TX) with the survey command. Population weights supplied by Statistics Canada were used to adjust for sampling probability and non-response and to ensure estimates were representative of the population of interest. As well, 1,000 bootstrap weights were applied to account for the impact of the systematic sampling approach on measures of variance around these estimates. More information on the methods used to produce the population and bootstrap weights is available elsewhere. ${ }^{21}$

Aspects of military to civilian transition that participants perceived as most challenging were identified using prevalence estimates for each type of perceived transition challenge among both medically released Veterans and those released under non-medical categories. Statistical significance between the groups was assessed by examining the $95 \%$ confidence intervals (CIs). These represent the range of values in which we can be $95 \%$ certain the true value lies within the population of interest. ${ }^{24}$ Non-overlapping CIs were assumed to indicate a significant difference. However, it should be noted that statistically significant differences may exist even when 95\% CIs overlap. In such instances, statistical tests should be conducted to assess statistical significance.

Logistic regression analyses were conducted to determine which perceived transition challenges were most strongly associated with difficult post-military adjustment. Pairs of items related to finding employment, identifying educational opportunities, and the loss of military identity were combined, leaving 7 types of challenges from the original 10.

Regression analyses were conducted to identify key correlates for the two transition challenges that were most strongly associated with difficult post-military adjustment (i.e., loss of military identity and financial preparedness). First, unadjusted logistic regression analyses were conducted to identify demographic and military characteristics that were statistically associated with the two transition challenges. These characteristics were then included as covariates in adjusted analyses conducted to examine the main and interactive effects of release type and self-rated health status on the two transition challenges. All possible main effects, two-way interactions, and the three-way interactions were examined. The margins command was used to help with the interpretation of interactions. This command generates predicted probabilities, which indicate the probability of a given outcome under different conditions. Specifically, these are calculated using the regression equation for that outcome by "plugging in" specified values for independent variables of interest (in this case, release type, self-rated physical health status, and self-rated mental health status).

\section{RESULTS}

Table 1 provides a summary of the characteristics of participants overall and by release type. Approximately half of the CAF Veterans were medically released (49.6\%), and the remaining half were released under nonmedical categories (50.4\%). Overall, they were primarily male and between the ages of 30 and 49 years. Regarding their military characteristics, the majority of participants had 20 years of service or more, were NCMs, and had primarily served in the army. About one-third reported poor health.

Noteworthy differences between medically released and non-medically released CAF Veterans included the apparent overrepresentation of women, NCMs, army 
Table 1. Participant characteristics, CAF Veterans released in 2016

\begin{tabular}{|c|c|c|c|c|c|c|c|c|c|}
\hline \multirow{3}{*}{ Variables } & \multicolumn{3}{|c|}{$\begin{array}{l}\text { Overall, weighted } \\
\qquad(N=4,100)\end{array}$} & \multicolumn{3}{|c|}{$\begin{array}{l}\text { Medical release, weighted } \\
\qquad(n=2,000)\end{array}$} & \multicolumn{3}{|c|}{$\begin{array}{l}\text { Non-medical release, } \\
\text { weighted }(n=2,100)\end{array}$} \\
\hline & \multirow[t]{2}{*}{$\%$} & \multicolumn{2}{|c|}{$95 \% \mathrm{Cl}$} & \multirow[t]{2}{*}{$\%$} & \multicolumn{2}{|c|}{$95 \% \mathrm{Cl}$} & \multirow[t]{2}{*}{$\%$} & \multicolumn{2}{|c|}{$95 \% \mathrm{Cl}$} \\
\hline & & LL & UL & & LL & UL & & LL & UL \\
\hline \multicolumn{10}{|l|}{ Sex } \\
\hline Male & 85.8 & 85.8 & 85.8 & 82.1 & 82.0 & 82.2 & 89.4 & 89.4 & 89.5 \\
\hline Female & 14.2 & 14.2 & 14.2 & 17.9 & 17.8 & 18.0 & 10.6 & 10.5 & 10.6 \\
\hline \multicolumn{10}{|l|}{ Age (years) } \\
\hline 20-29 & 17 & 15.6 & 18.5 & 9.7 & 8.3 & 11.4 & 24.2 & 21.8 & 26.7 \\
\hline 30-39 & 25.1 & 23.7 & 26.6 & 27.6 & 26.1 & 29.2 & 22.6 & 20.3 & 25.1 \\
\hline $40-49$ & 26.4 & 24.6 & 28.4 & 33.0 & 30.3 & 35.8 & 19.9 & 17.6 & 22.5 \\
\hline $50+$ & 31.5 & 29.6 & 33.4 & 29.6 & 26.9 & 32.4 & 33.4 & 30.9 & 35.9 \\
\hline \multicolumn{10}{|l|}{ Marital status } \\
\hline Single & 21.8 & 20.1 & 23.6 & 17.5 & 15.3 & 19.8 & 26.1 & 23.5 & 29.0 \\
\hline Married/common law & 67 & 64.9 & 69 & 66.8 & 63.9 & 69.6 & 67.2 & 64.0 & 70.2 \\
\hline Formerly married & 11.2 & 9.8 & 12.7 & 15.8 & 13.6 & 18.2 & 6.7 & 5.2 & 8.6 \\
\hline \multicolumn{10}{|l|}{ First official language } \\
\hline English & 70.4 & 68.1 & 72.6 & 67.6 & 64.6 & 70.5 & 73.1 & 69.7 & 76.3 \\
\hline French & 29.6 & 27.4 & 31.9 & 32.4 & 29.5 & 35.4 & 26.9 & 23.7 & 30.3 \\
\hline \multicolumn{10}{|l|}{ Education } \\
\hline Up to high school & 43.5 & 41.2 & 45.9 & 45.3 & 42.0 & 48.5 & 41.8 & 38.4 & 45.3 \\
\hline Trade/college & 31.3 & 29.1 & 33.6 & 35.3 & 32.3 & 38.4 & 27.4 & 24.3 & 30.8 \\
\hline At least some university & 25.2 & 23.1 & 27.3 & 19.5 & 17.0 & 22.2 & 30.7 & 27.6 & 34.1 \\
\hline \multicolumn{10}{|l|}{ Years of service } \\
\hline up to 5 & 9.1 & 7.9 & 10.4 & 4.1 & 3.1 & 5.3 & 14.1 & 11.9 & 16.5 \\
\hline $6-9$ & 15.7 & 14.2 & 17.3 & 11.4 & 9.8 & 13.3 & 19.8 & 17.4 & 22.5 \\
\hline $10-19$ & 24.2 & 22.4 & 26 & 33.1 & 30.5 & 35.8 & 15.4 & 13.2 & 18.0 \\
\hline $20-34$ & 39.6 & 37.8 & 41.5 & 41.4 & 38.7 & 44.1 & 37.9 & 35.4 & 40.4 \\
\hline $35+$ & 11.4 & 10 & 13 & 10.1 & 8.2 & 12.3 & 12.8 & 10.8 & 15.2 \\
\hline \multicolumn{10}{|l|}{ Rank } \\
\hline Junior NCM & 46.1 & 44.2 & 48.1 & 49.7 & 46.8 & 52.5 & 42.7 & 40.0 & 45.4 \\
\hline Senior NCM & 31.5 & 29.6 & 33.6 & 34.9 & 32.1 & 37.7 & 28.2 & 25.4 & 31.2 \\
\hline Junior officer & 10.7 & 9.3 & 12.2 & 9.6 & 8.0 & 11.5 & 11.8 & 9.7 & 14.3 \\
\hline Senior officer & 11.6 & 10.2 & 13.3 & 5.9 & 4.5 & 7.6 & 17.3 & 14.8 & 20.1 \\
\hline \multicolumn{10}{|l|}{ Environment } \\
\hline Sea & 16.2 & 14.4 & 18.2 & 12.4 & 10.5 & 14.7 & 19.9 & 17.2 & 23.0 \\
\hline Land & 57.2 & 55 & 59.4 & 65.9 & 62.8 & 68.9 & 48.7 & 45.3 & 52.0 \\
\hline Air & 26.6 & 24.4 & 28.9 & 21.7 & 19.1 & 24.5 & 31.4 & 28.1 & 34.9 \\
\hline \multicolumn{10}{|l|}{ Afghanistan deployment } \\
\hline Yes & 48.9 & 51.1 & 51.1 & 59.0 & 55.9 & 62.1 & 39.0 & 36.0 & 42.1 \\
\hline No & 51.1 & 53.2 & 53.2 & 41.0 & 37.9 & 44.1 & 61.0 & 57.9 & 64.0 \\
\hline \multicolumn{10}{|l|}{ Other deployment } \\
\hline Yes & 55.3 & 53.2 & 57.3 & 56.3 & 53.4 & 59.2 & 54.2 & 51.4 & 57.1 \\
\hline No & 44.7 & 42.7 & 46.8 & 43.7 & 40.8 & 46.6 & 45.8 & 42.9 & 48.6 \\
\hline
\end{tabular}

(Continued) 
Table 1. (Continued)

\begin{tabular}{|c|c|c|c|c|c|c|c|c|c|}
\hline \multirow{3}{*}{ Variables } & \multicolumn{3}{|c|}{$\begin{array}{l}\text { Overall, weighted } \\
\qquad(N=4,100)\end{array}$} & \multicolumn{3}{|c|}{$\begin{array}{l}\text { Medical release, weighted } \\
(n=2,000)\end{array}$} & \multicolumn{3}{|c|}{$\begin{array}{l}\text { Non-medical release, } \\
\text { weighted }(n=2,100)\end{array}$} \\
\hline & \multirow[t]{2}{*}{$\%$} & \multicolumn{2}{|c|}{$95 \% \mathrm{Cl}$} & \multirow[t]{2}{*}{$\%$} & \multicolumn{2}{|c|}{$95 \% \mathrm{Cl}$} & \multirow[t]{2}{*}{$\%$} & \multicolumn{2}{|c|}{$95 \% \mathrm{Cl}$} \\
\hline & & LL & UL & & LL & UL & & LL & UL \\
\hline \multicolumn{10}{|l|}{ Physical health status } \\
\hline Poor/fair & 33.0 & 31.2 & 34.9 & 57.6 & 54.5 & 60.7 & 8.8 & 7.0 & 10.9 \\
\hline Good & 29.9 & 27.8 & 32.1 & 30.9 & 28.1 & 33.9 & 28.9 & 25.8 & 32.1 \\
\hline Very good/excellent & 37.1 & 35.1 & 39.1 & 11.4 & 9.6 & 13.5 & 62.4 & 58.9 & 65.7 \\
\hline \multicolumn{10}{|l|}{ Mental health status } \\
\hline Poor/fair & 32.2 & 30.3 & 34.2 & 54.4 & 51.1 & 57.6 & 10.4 & 8.5 & 12.7 \\
\hline Good & 22.4 & 20.5 & 24.5 & 25.3 & 22.6 & 28.3 & 19.6 & 17.0 & 22.5 \\
\hline Very good/excellent & 45.3 & 43.2 & 47.4 & 20.3 & 17.9 & 23.0 & 70.0 & 66.5 & 73.3 \\
\hline
\end{tabular}

Table 2. Results of logistic regression analyses of the associations of perceived challenges with difficult post-military adjustment

\begin{tabular}{|c|c|c|c|c|c|c|c|c|}
\hline \multirow[t]{2}{*}{ Perceived challenge } & \multirow[t]{2}{*}{ OR } & \multicolumn{2}{|c|}{$95 \% \mathrm{Cl}$} & \multirow[t]{2}{*}{$p$} & \multirow[t]{2}{*}{ AOR } & \multicolumn{2}{|c|}{$95 \% \mathrm{Cl}$} & \multirow[t]{2}{*}{$p$} \\
\hline & & $\mathrm{LL}$ & UL & & & LL & UL & \\
\hline Being financially prepared & 4.05 & 3.19 & 5.13 & $<0.001$ & 2.32 & 1.76 & 3.06 & $<0.001$ \\
\hline Finding employment & 3.94 & 3.07 & 5.06 & $<0.001$ & 1.89 & 1.39 & 2.56 & $<0.001$ \\
\hline Finding educational opportunities & 3.9 & 2.96 & 5.16 & $<0.001$ & 1.73 & 1.23 & 2.45 & 0.002 \\
\hline Relocating & 2.81 & 2.1 & 3.76 & $<0.001$ & 1.54 & 1.07 & 2.21 & 0.02 \\
\hline Finding a health care provider & 2.51 & 2.06 & 3.06 & $<0.001$ & 1.99 & 1.58 & 2.5 & $<0.001$ \\
\hline Understanding benefits & 2.48 & 2.01 & 3.07 & $<0.001$ & 1.38 & 1.07 & 1.79 & 0.014 \\
\hline Losing military identity & 7.51 & 6.04 & 9.33 & $<0.001$ & 5.36 & 4.26 & 6.74 & $<0.001$ \\
\hline
\end{tabular}

$\mathrm{OR}=$ odds ratio; $\mathrm{Cl}=$ confidence interval; $\mathrm{AOR}$ = adjusted odds ratio; $\mathrm{LL}=$ lower limit; $\mathrm{UL}$ = upper limit.

personnel, and individuals with a history of deployment in Afghanistan among those who were medically released, relative to those who were non-medically released. As well, a much higher proportion of medically released CAF Veterans reported poor health.

\section{Perceived transition challenges}

Figure 1 shows the proportions of CAF Veterans who perceived each item as challenging during their transition, by release type. Although all of the perceived transition challenges were more prevalent among medically released Veterans, challenges related to finding a health care provider, losing one's military identity, understanding available services and benefits, losing touch with military peers, and being financially prepared were most prevalent, regardless of release type.

Table 2 shows the results of unadjusted and adjusted regression models for difficult post-military adjustment. All seven perceived transition challenges were significantly associated with difficult post-military adjustment. However, the association was strongest for loss of military identity (adjusted odds ratio $[\mathrm{AOR}]=5.4$ ). Also, Veterans who perceived financial preparedness as a challenge had about twice the odds of reporting difficult adjustment $(\mathrm{AOR}=2.3)$. Therefore, subsequent analyses focused on identifying correlates of perceived transition challenges related to loss of military identity and financial preparedness.

\section{Correlates of perceived transition challenges}

Table 3 shows the results of unadjusted logistic regression models for the perceived transition challenges. Veterans who were medically released, or who reported poor physical or mental health, had substantially greater odds of perceiving the loss of military identity or financial preparedness as challenges. The associations were strongest for loss of military identity. Regarding 
Table 3. Results of unadjusted logistic regression analyses of the associations of demographic/military characteristics with perceived transition challenges

\begin{tabular}{|c|c|c|c|c|c|c|c|c|}
\hline \multirow[t]{3}{*}{ Predictor } & \multicolumn{4}{|c|}{ Loss of military identity } & \multicolumn{4}{|c|}{ Financial preparedness } \\
\hline & \multirow[t]{2}{*}{ OR } & \multicolumn{2}{|c|}{$95 \% \mathrm{Cl}$} & \multirow[t]{2}{*}{$p$} & \multirow[t]{2}{*}{ OR } & \multicolumn{2}{|c|}{$95 \% \mathrm{Cl}$} & \multirow[t]{2}{*}{$p$} \\
\hline & & LL & UL & & & LL & UL & \\
\hline \multicolumn{9}{|l|}{ Age (years) } \\
\hline 20-29 (Ref.) & - & & & & - & & & \\
\hline $30-39$ & 1.62 & 1.21 & 2.18 & 0.001 & 1.76 & 1.21 & 2.55 & 0.003 \\
\hline $40-49$ & 1.29 & 0.96 & 1.75 & 0.094 & 1.39 & 0.94 & 2.05 & 0.096 \\
\hline $50+$ & 0.57 & 0.42 & 0.77 & $<0.001$ & 0.72 & 0.49 & 1.07 & 0.102 \\
\hline \multicolumn{9}{|l|}{ Sex } \\
\hline Men (Ref.) & - & & & & - & & & \\
\hline Women & 1.64 & 1.4 & 1.92 & $<0.001$ & 1.4 & 1.16 & 1.68 & $<0.001$ \\
\hline \multicolumn{9}{|l|}{ Rank } \\
\hline Junior NCM (Ref.) & - & & & & - & & & \\
\hline Senior NCM & 0.59 & 0.47 & 0.74 & $<0.001$ & 0.53 & 0.4 & 0.71 & $<0.001$ \\
\hline Junior Officer & 0.48 & 0.35 & 0.67 & $<0.001$ & 0.51 & 0.34 & 0.75 & 0.001 \\
\hline Senior Officer & 0.26 & 0.17 & 0.38 & $<0.001$ & 0.22 & 0.13 & 0.37 & $<0.001$ \\
\hline \multicolumn{9}{|l|}{ Element } \\
\hline Army (Ref.) & - & & & & - & & & \\
\hline Navy & 0.33 & 0.25 & 0.44 & $<0.001$ & 0.82 & 0.58 & 1.14 & 0.234 \\
\hline Air Force & 0.52 & 0.41 & 0.64 & $<0.001$ & 0.69 & 0.53 & 0.9 & 0.006 \\
\hline \multicolumn{9}{|c|}{ Deployed to Afghanistan } \\
\hline No (Ref.) & - & & & & - & & & \\
\hline Yes & 1.42 & 1.16 & 1.73 & 0.001 & 1.22 & 0.97 & 1.53 & 0.091 \\
\hline \multicolumn{9}{|c|}{ Deployed outside North America } \\
\hline No (Ref.) & - & & & & - & & & \\
\hline Yes & 0.67 & 0.55 & 0.81 & $<0.001$ & 0.81 & 0.64 & 1.02 & 0.073 \\
\hline \multicolumn{9}{|l|}{ Years of service } \\
\hline 2-5 (Ref.) & - & & & & - & & & \\
\hline $6-9$ & 1.98 & 1.28 & 3.05 & 0.002 & 1.79 & 1.06 & 3.03 & 0.028 \\
\hline $10-19$ & 2.79 & 1.88 & 4.12 & $<0.001$ & 2.46 & 1.53 & 3.94 & $<0.001$ \\
\hline $20-34$ & 1.01 & 0.69 & 1.5 & 0.942 & 1.16 & 0.72 & 1.89 & 0.539 \\
\hline $35+$ & 0.87 & 0.54 & 1.41 & 0.583 & 0.74 & 0.4 & 1.4 & 0.356 \\
\hline \multicolumn{9}{|l|}{ Release type } \\
\hline Non-medical (Ref.) & - & & & & - & & & \\
\hline Medical & 4.5 & 3.63 & 5.57 & $<0.001$ & 2.97 & 2.3 & 3.84 & $<0.001$ \\
\hline \multicolumn{9}{|l|}{ Physical health } \\
\hline Good (Ref.) & - & & & & - & & & \\
\hline Poor & 3.64 & 2.96 & 4.48 & $<0.001$ & 2.95 & 2.33 & 3.73 & $<0.001$ \\
\hline \multicolumn{9}{|l|}{ Mental health } \\
\hline Good (Ref.) & - & & & & - & & & \\
\hline Poor & 6.4 & 5.12 & 8 & $<0.001$ & 3.67 & 2.91 & 4.61 & $<0.001$ \\
\hline
\end{tabular}

$\mathrm{OR}$ = odds ratio; $\mathrm{Cl}=$ confidence interval; $\mathrm{LL}$ = lower limit; $\mathrm{UL}=$ upper limit; Ref. = reference category; $\mathrm{NCM}=$ noncommissioned member. 
demographic and military characteristics, the odds of perceiving loss of military identity as a challenge were highest among Veterans who were aged 30-39 years, female, junior NCM, primarily in the army, deployed in support of the Afghanistan mission, or had between 6 and 19 years of service. Conversely, Veterans who were more than 50 years old and were deployed in support of another mission outside of North America demonstrated lower odds of perceiving loss of military identity as a challenge. The odds of perceiving financial preparedness as a challenge were highest among Veterans who were 30-39 years of age, female, junior NCMs, primarily in the army, or had between 6 and 19 years of service relative to their referent counterparts. Given that each of the characteristics was associated with at least one of the perceived transition challenges, all were included in subsequent adjusted analyses as covariates.

Table 4 shows the results of the adjusted regression models for the perceived transition challenges. Adjusting for the covariates, the odds of perceiving loss of military identity as a challenge were significantly greater among Veterans who were medically released, reported poor physical health, and reported poor mental health (holding all other variables in the interaction at the reference value). However, no significant interactions were observed among these three variables.

Adjusting for the covariates, the odds of perceiving financial preparedness as a challenge were highest among Veterans who were medically released, reported poor physical health, and reported poor mental health. Significant interactions were observed between release type and mental health status, physical and mental health status, and between release, physical health, and mental health status. Figure 2 better illustrates the results of the interactive effects of these variables using the predictive probabilities calculated based on the resulting regression equation for perceiving financial preparedness as a challenge within each of the release, physical health, and mental health subgroups of Veterans. Veterans with poor mental health had lower odds of perceiving financial preparedness as a challenge if they were medically released (illustrated in Figure 2 with bars comparing

Table 4. Results of adjusted logistic regressions of the associations of demographic/military characteristics, medical release, and health status with perceived transition challenges

\begin{tabular}{|c|c|c|c|c|c|c|c|c|}
\hline \multirow[t]{3}{*}{ Predictor } & \multicolumn{4}{|c|}{ Loss of military identity } & \multicolumn{4}{|c|}{ Financial preparedness } \\
\hline & \multirow[t]{2}{*}{ AOR } & \multicolumn{2}{|c|}{$95 \% \mathrm{Cl}$} & \multirow[t]{2}{*}{$p$} & \multirow[t]{2}{*}{ AOR } & \multicolumn{2}{|c|}{$95 \% \mathrm{Cl}$} & \multirow[t]{2}{*}{$p$} \\
\hline & & $\mathrm{LL}$ & UL & & & LL & UL & \\
\hline \multicolumn{9}{|l|}{ Age (years) } \\
\hline 20-29 (Ref.) & - & & & & - & & & \\
\hline $30-39$ & 1.14 & 0.76 & 1.71 & 0.52 & 1.31 & 0.84 & 2.03 & 0.238 \\
\hline $40-49$ & 1.17 & 0.69 & 2 & 0.563 & 1.32 & 0.76 & 2.33 & 0.319 \\
\hline $50+$ & 0.68 & 0.37 & 1.22 & 0.191 & 0.93 & 0.51 & 1.69 & 0.802 \\
\hline \multicolumn{9}{|l|}{ Sex } \\
\hline Men (Ref.) & - & & & & - & & & \\
\hline Women & 1.53 & 1.23 & 1.92 & $<0.001$ & 1.17 & 0.93 & 1.48 & 0.175 \\
\hline \multicolumn{9}{|l|}{ Rank } \\
\hline Junior NCM (Ref.) & - & & & & - & & & \\
\hline Senior NCM & 0.94 & 0.65 & 1.35 & 0.741 & 0.59 & 0.4 & 0.86 & 0.007 \\
\hline Junior Officer & 0.65 & 0.44 & 0.94 & 0.023 & 0.6 & 0.4 & 0.89 & 0.012 \\
\hline Senior Officer & 0.62 & 0.38 & 1.03 & 0.066 & 0.34 & 0.19 & 0.6 & 0 \\
\hline \multicolumn{9}{|l|}{ Element } \\
\hline Army (Ref.) & - & & & & - & & & \\
\hline Navy & 0.45 & 0.32 & 0.63 & $<0.001$ & 1.16 & 0.79 & 1.69 & 0.454 \\
\hline Air Force & 0.71 & 0.54 & 0.93 & 0.015 & 0.95 & 0.7 & 1.3 & 0.75 \\
\hline \multicolumn{9}{|c|}{ Deployed to Afghanistan } \\
\hline No (Ref.) & - & & & & - & & & \\
\hline Yes & 0.98 & 0.74 & 1.28 & 0.862 & 0.96 & 0.71 & 1.29 & 0.785 \\
\hline
\end{tabular}


Table 4. (Continued)

\begin{tabular}{|c|c|c|c|c|c|c|c|c|}
\hline \multirow{3}{*}{ Predictor } & \multicolumn{4}{|c|}{ Loss of military identity } & \multicolumn{4}{|c|}{ Financial preparedness } \\
\hline & \multirow[t]{2}{*}{ AOR } & \multicolumn{2}{|c|}{$95 \% \mathrm{Cl}$} & \multirow[t]{2}{*}{$p$} & \multirow[t]{2}{*}{ AOR } & \multicolumn{2}{|c|}{$95 \% \mathrm{Cl}$} & \multirow[t]{2}{*}{$p$} \\
\hline & & LL & UL & & & LL & UL & \\
\hline \multicolumn{9}{|l|}{ Deployed outside North America } \\
\hline No (Ref.) & - & & & & - & & & \\
\hline Yes & 0.95 & 0.72 & 1.26 & 0.738 & 1.12 & 0.82 & 1.52 & 0.474 \\
\hline \multicolumn{9}{|l|}{ Years of service } \\
\hline 2-5 (Ref.) & - & & & & - & & & \\
\hline $2-9$ & 1.7 & 1.04 & 2.77 & 0.033 & 1.64 & 0.92 & 2.92 & 0.091 \\
\hline $10-19$ & 1.52 & 0.9 & 2.55 & 0.116 & 1.64 & 0.89 & 3.02 & 0.11 \\
\hline $20-34$ & 0.92 & 0.48 & 1.8 & 0.818 & 1.32 & 0.63 & 2.78 & 0.461 \\
\hline $35+$ & 1.57 & 0.72 & 3.41 & 0.258 & 1.25 & 0.51 & 3.03 & 0.626 \\
\hline \multicolumn{9}{|l|}{ Release type $^{\star}$} \\
\hline Non-medical (Ref.) & - & & & & - & & & \\
\hline Medical & 2.06 & 1.48 & 2.86 & $<0.001$ & 2.18 & 1.4 & 3.4 & 0.001 \\
\hline \multicolumn{9}{|l|}{ Physical health $^{\dagger}$} \\
\hline Good (Ref.) & - & & & & - & & & \\
\hline Poor & 2.35 & 1.12 & 4.93 & 0.024 & 2.87 & 1.27 & 6.46 & 0.001 \\
\hline \multicolumn{9}{|l|}{ Mental health ${ }^{\ddagger}$} \\
\hline Good (Ref.) & - & & & & - & & & \\
\hline Poor & 4.23 & 2.23 & 8.05 & $<0.001$ & 5.24 & 2.74 & 10.01 & $<0.001$ \\
\hline Release $\times$ Physical health ${ }^{\S}$ & 0.69 & 0.29 & 1.63 & 0.396 & 0.39 & 0.15 & 1.03 & 0.058 \\
\hline Release $\times$ Mental health" & 1.17 & 0.53 & 2.58 & 0.705 & 0.25 & 0.11 & 0.56 & 0.001 \\
\hline Physical health $\times$ Mental health ${ }^{\star \star}$ & 0.34 & 0.1 & 1.16 & 0.085 & 0.22 & 0.06 & 0.86 & 0.029 \\
\hline Release $\times$ Physical health $\times$ Mental health ${ }^{\dagger \dagger}$ & 1.62 & 0.39 & 6.64 & 0.506 & 7.44 & 1.62 & 34.06 & 0.01 \\
\hline
\end{tabular}

$\mathrm{AOR}$ = adjusted odds ratio; $\mathrm{Cl}=$ confidence interval; $\mathrm{LL}=$ lower limit; $\mathrm{UL}=$ upper limit; Ref. = reference category; $\mathrm{NCM}=$ non-commissioned member.

* Effect of medical release when physical and mental health are good.

$\dagger$ Effect of poor physical health among non-medically released with good mental health.

$\ddagger$ Effect of poor mental health among non-medically released with good physical health.

$\S$ Effect of medical release when only physical health is poor.

I Effect of medical release when only mental health is poor.

** Effect of poor physical health among non-medically released with poor mental health.

†† Effect of medical release when physical and mental health are poor.

non-medically and medically released Veterans with poor mental health only). Non-medically released Veterans with poor mental health also had lower odds of perceiving financial preparedness as a challenge if their physical health was also poor (illustrated in Figure 2 by comparing light grey bars for those with poor mental health only versus poor physical/mental health). Results of the significant three-way interaction underlined substantially greater odds of perceiving financial preparedness as a challenge $(\mathrm{AOR}=7.44)$ among medically released Veterans with poor physical/mental health compared to non-medically released Veterans with good physical/mental health (illustrated in Figure 2 with bars comparing non-medically released Veterans with good physical/mental health and medically released Veterans with poor physical/mental health).

\section{DISCUSSION}

The present study extends prior research by providing more information on the nature, extent, and correlates of perceived military to civilian transition challenges in a representative sample of newly released CAF Veterans. Challenges were reported by all Veterans but were found to be more prevalent among medically released 


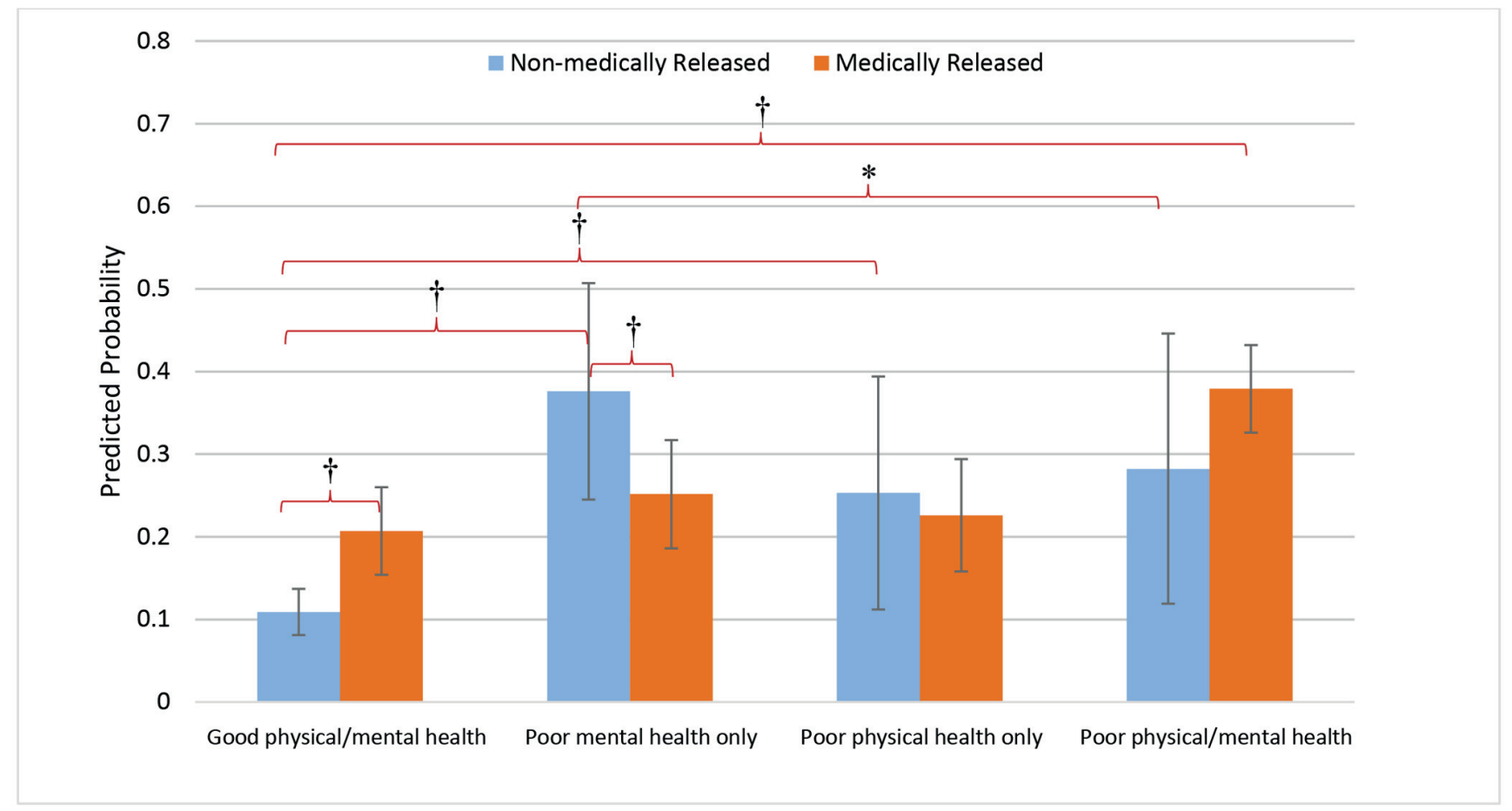

Figure 2. Predicted probabilities for perceived transition challenges related to financial preparedness

${ }^{*} p<0.01$

$\dagger p<0.001$

Veterans. All were independently associated with difficult post-military adjustment, although perceived loss of military identity and challenges with financial preparedness had the strongest associations. Further regression analyses identified subgroups in whom loss of military identity and challenges with financial preparedness were more common. Although being medically released was strongly associated with reporting both types of challenges, so were poor self-rated physical health, poor self-rated mental health, and a range of other sociodemographic and military characteristics, independently of release type.

\section{Loss of military identity}

Among all of the perceived transition challenges, loss of military identity had the strongest association with difficult post-military adjustment. Social identity challenges in adjustment to post-military culture have been proposed as a defining aspect of military to civilian transition. ${ }^{25}$ Initially formed through socialization during recruit training, military identity tends to be strong among service members throughout their careers. After military service, intimate connections to military culture are lost, and service members must develop new social identities by finding meaning through memberships in social groups whose norms and values they learn to value. Loss of military identity, and the failure to achieve a new post-military social identity, have been found to be major barriers to successful transition. ${ }^{1,15,26}$

Relative to men, women were more likely to perceive loss of military identity as a challenge. Several unique aspects of women's military experiences may have contributed to this. Representing only $15 \%$ of the CAF population, women are typically employed in only a small number of military occupation groups. Women in the CAF have also been found to be at greater risk of experiencing assault or discrimination on the basis of their sex..$^{27,28}$ In combination, these shared aspects of the military experience may contribute to particularly strong bonds among women in the CAF, resulting in the potential for a greater sense of loss if they are broken. Furthermore, it has been proposed that one way in which women adjust to the military is by behaving like "one of the guys" and working harder than their male peers to prove themselves. Over time, female members may become more masculine in order to fit in and be perceived as able to do the work of a soldier. ${ }^{29}$ For female Veterans, re-entering the civilian world is "further complicated by 
the need to transition from being a soldier - an identity almost devoid of femaleness - to being a civilian and negotiating what it means to be female." 30 ( p.506) Consequently, a strong military identity may make it hard for female Veterans to assume a traditional civilian female identity, leading them to feel isolated..$^{30}$

Veterans who were medically released, as well as those who reported poor physical or mental health regardless of their release category, were also more likely to perceive loss of military identity as a challenge relative to their respective counterparts. Indeed, for ill or injured Veterans, the transition process may also involve having to negotiate the concept of oneself as disabled. ${ }^{31}$ As well, members who are released with health problems, either medically or non-medically, may have been released before they were ready to leave their military lives behind.

\section{Financial preparedness}

Financial well-being has been found to be associated with various aspects of mental and physical well-being in military families ${ }^{32}$ in addition to Veterans' post-military adjustment. ${ }^{33-35}$ Furthermore, there is evidence many CAF members are not sufficiently financially prepared for release. ${ }^{36}$ Consistent with the LASS surveys, results indicated that financial preparedness challenges were especially common among junior NCMs. ${ }^{33}$ While their lower pension may have contributed to this, uncertainty regarding future income sources may also have played a role. Junior NCMs have been found to be more likely to report lower transferability of their skills, ${ }^{37}$ which may impact employability. They have also been found to be less knowledgeable about their pensions and to report more uncertainty in its ability to support them financially in retirement. ${ }^{36}$

Veterans' release type was also found to be associated with perceived financial preparedness challenges, although some noteworthy interaction effects were observed when health status was considered. Among Veterans with poor self-rated mental health, those who were medically released were less - rather than more likely to perceive financial preparedness as a challenge. It is possible enhanced access to benefits gained through the medical release process helped mitigate financial strain among Veterans with poor mental health. Why this did not appear to be the case for Veterans with poor self-rated physical health remains unclear. One possibility is that freely available health care more readily addressed their needs, resulting in fewer costs.

\section{Strengths and limitations}

Since the survey was cross-sectional, no inferences can be made on causality. However, a major strength of the present study entails its basis on a representative sample of newly released CAF Veterans. While the structured nature of the survey did not permit the exploration and identification of emerging issues related to military to civilian transition, its quantitative findings are generally consistent with past qualitative studies ${ }^{1,19,20,35}$ and provide converging evidence on the importance of transition challenges and their impacts on well-being. Finally, only single-item measures of self-rated physical and mental health were examined. Such measures have been found to be highly correlated with morbidity, disability, service utilization, and multi-item measures of health. ${ }^{38-40}$ Still, further analysis of the CAFTWS is needed to explore the health conditions of newly released CAF Veterans in greater detail.

\section{Implications}

By underlining the importance of loss of military identity, this study supports recommendations that social identity challenges should be addressed during military to civilian transition. ${ }^{15-17,25}$ Unlike programs and services that provide service members and Veterans with more tangible forms of support (e.g., financial support), programs and services that can address existential transition challenges of a more psychosocial nature are relatively less well-established. Achievement of post-military social identities that connect Veterans with needed well-being resources is integral to post-military adjustment. ${ }^{41}$ The Veterans' Identities Research Theme Working Group generated a number of recommendations for doing so, including preparing service members prior to their transition to actively manage their social identity challenges, enabling peer support during transition, asking Veterans what forms of commemoration and recognition work for them, and sensitizing civilian communities to take active roles in embracing transitioning Veterans and their families. ${ }^{17,25}$ Additional research on issues around social identity during military to civilian transition might include the further development of relevant measures and evaluation of approaches for addressing such challenges.

Findings regarding the magnitude of perceived challenges with financial preparedness underline the value of financial counselling or training programs, such as those offered by the Service Income Security Insurance Plan (SISIP) and various financial support 
benefits that are currently available to support Veterans. ${ }^{42}$ Although CAF members are encouraged to seek these services in the early stages of their retirement planning, lower-ranking members, or those who are released unexpectedly due to health problems, may be less likely to do so. Again, further research should focus on identifying the best means to empower CAF members early in their military career to plan for their financial future.

\section{Conclusion}

Consistent with findings published in both Canada and elsewhere, ${ }^{3,4,13,15,16}$ this study found that both medically and non-medically released Veterans perceive a variety of challenges during military to civilian transition. This study not only demonstrates the potential value of enhancing Veterans' preparedness for loss of military identity and managing finances, but also for finding education and employment opportunities, relocating, finding health care providers, and navigating the vast amount of information they will receive on relevant benefits and services. It is important to note that significant efforts have been made to enhance transition program services in the CAF since the CAFTWS was administered. As such, results provide valuable baseline information against which progress related to these efforts can be monitored.

\section{REFERENCES}

1. Black T, Papile C. Making it on civvy street: an online survey of Canadian Veterans in transition. Can J Couns Psychother. 2010;44(4):383-401. https://psycnet.apa. org/record/2012-14112-004.

2. MacLean MB, Van Til L, Thompson JM, et al. Postmilitary adjustment to civilian life: potential risks and protective factors. Phys Ther. 2014;94(8):1186-95. https://doi.org/10.2522/ptj.20120107. Medline:23766397

3. Vogt D, Perkins DF, Copeland LA, et al. The Veterans Metrics Initiative study of US Veterans' experiences during their transition from military service. BMJ Open. 2018;8(6):e020734. https://doi.org/10.1136/ bmjopen-2017-020734. Medline:29895650

4. Pedlar D, Thompson JM, Castro CA. Military to civilian transition theories and frameworks. In: Castro CA, Dursun S, editors. Military Veteran reintegration: approach, management, and assessment of military Veterans transitioning to civilian life. San Diego, CA: Elsevier Academic Press; 2019. p. 24-51.

5. Thompson JM, MacLean MB, Roach MB, et al. A well-being construct for Veterans' policy, programming and research. Charlottetown, PE: Research Directorate, Veterans Affairs Canada; 2016.
6. Department of National Defence [homepage on the Internet]. Well-being framework. Ottawa, ON: Government of Canada; 2019 [updated 2019 Nov 6; cited 2020 Mar 27]. Available from: https://www.canada. ca/en/department-national-defence/corporate/reports-publications/transition-guide/well-being-framework.html.

7. Thompson JM, Van Til L, Poirier A, et al. Health and well-being of Canadian Armed Forces Veterans: findings from the 2013 Life After Service Survey. Research Directorate Technical Report. Charlottetown, PE: Research Directorate, Veterans Affairs Canada; 2014.

8. Van Til L, Sweet J, Poirier A, et al. Well-being of Canadian regular force Veterans: findings from LASS 2016 survey. Research Directorate Technical Report. Charlottetown, PE: Research Directorate, Veterans Affairs Canada; 2017.

9. Tweel M, Thompson JM, Lockhart W, et al. Veterans Affairs Canada Research Directorate publications: annotated bibliography 1997-2018. Charlottetown, PE: Research Directorate, Veterans Affairs Canada; 2019.

10. Auditor General of Canada. 2012 fall report of the Auditor General of Canada. Ottawa, ON: Auditor General of Canada; 2012.

11. VanTil L, Macintosh S, Thompson J, et al. Fast facts on Veterans' transition experiences. J Mil Vet Fam Health. 2015;1(1):7-8. https://doi.org/10.3138/jmvfh.1.1.7.

12. Hachey KK, Sudom K, Sweet J, et al. Transitioning from military to civilian life: the role of mastery and social support. J Mil Vet Fam Health. 2016;2(1):9-18. https://doi.org/10.3138/jmvfh.3379.

13. Thompson JM, Sweet J, Van Til L, et al. Correlates of mental health problems in Canadian Armed Forces Veterans - 2013 Life After Service Survey. Research Directorate Technical Report. Charlottetown, PE: Research Directorate, Veterans Affairs Canada; 2016 Sep 14. Available from: https://cimvhr.ca/vac-reports/data/ reports/Thompson\%202016_Correlates\%20of\%20 Mental\%20Health\%20Problems\%20in\%20CAF\%20 Veterans\%20LASS\%202013.pdf.

14. Thompson JM, Dursun S, Van Til L, et al. Group identity, difficult adjustment to civilian life and suicidal ideation in Canadian Armed Forces Veterans: Life After Service Studies. J Mil Vet Fam Health. 2019;5(2):10014. https://doi.org/10.3138/jmvfh.2018-0038.

15. Orazem RJ, Frazier PA, Schnurr PP, et al. Identity adjustment among Afghanistan and Iraq war Veterans with reintegration difficulty. Psychol Trauma. 2017;9(Suppl 1):4-11. https://doi.org/10.1037/ tra0000225. Medline:27893267

16. Keeling M. Stories of transition: narratives of US Veteran' military to civilian transition. J Mil Vet Fam Health. 2018;4(2):28-36. https://doi.org/10.3138/ jmvfh.2017-0009. 
17. Thompson JM, Lockhart W, Roach MB, et al. Veterans' identities and well-being in transition to civilian life: a resource for policy analysts, program designers, service providers and researchers. Report of the Veterans' Identities Research Theme Working Group. Charlottetown, PE: Veterans Affairs Canada; 2017.

18. Van Til L, Fikretoglu D, Pranger T, et al. Work reintegration for Veterans with mental disorders: a systematic literature review to inform research. Phys Ther. 2013;93(9):1163-74. https://doi.org/10.2522/ ptj.20120156. Medline:23043148

19. Blackburn D. Out of uniform: psychosocial issues experienced and coping mechanisms used by Veterans during the military-civilian transition. J Mil Vet Fam Health. 2017;3(1):62-9. https://doi.org/10.3138/ jmvfh.4160.

20. Cathcart D. Mandatory career change: transition experiences of Canadian armed forces Veterans [thesis]. Kingston, ON: Queen's University; 2018.

21. Statistics Canada [homepage on the Internet]. Canadian Armed Forces transition and well-being survey (CAFTWS). Ottawa, ON: Statistics Canada; 2018 [updated 2018 Mar 13; cited 2019 Dec 1]. Available from: https://www23.statcan.gc.ca/imdb/p2SV.pl?Function $=$ getSurvInstrumentList $\& I d=359214$.

22. Lee JEC, Skomorovsky A, Martynova E, et al. Pilot study on the well-being of ill or injured CAF members and their families: results of descriptive analyses and implications for future research. (Report DRDC-RDDC2016-R263). Ottawa, ON: Government of Canada; 2016.

23. Goldberg B. Military family update. Presented at AMBA Fall Workshop 2015; 2015 Aug 31-Sep 2; Leesburg, Virginia. Washington, DC: Association of Military Banks of America (AMBA); 2015.

24. Gerstman BB. Basics of confidence intervals. In: Gerstman BB, editor. Basic biostatistics: statistics for public health practice. Sudbury, MA: Jones and Bartlett Publishers, Inc; 2008. p. 197-208.

25. Atuel H, Castro CA. Military transition process and Veteran identity. In: Weiss EL, Castro CA, editors. American military life in the 21st century: social, cultural, and economic issues and trends. Santa Barbara, CA: ABC-CLIO; 2019. p. 485-96.

26. Grimell J. Self-reorganization in transition from military to civilian life: Maria's way. Ment Health Fam Med. 2017;13:544-53.

27. Cotter A. Sexual misconduct in the Canadian Armed Forces, 2016 [Internet]. Ottawa, ON: Statistics Canada; 2016 [updated 2016 Nov 28; cited 2020 Mar 27]. Available from: https://www150.statcan.gc.ca/n1/ $\mathrm{pub} / 85-603-\mathrm{x} / 85-603-\mathrm{x} 2016001$-eng.htm.

28. Watkins K, Bennett R, Zamorski MA, et al. Militaryrelated sexual assault in Canada: a cross-sectional survey. CMAJ Open. 2017;5(2):E496-E507. https:// doi.org/10.9778/cmajo.20160140. Medline:28642253

29. Waruszynski BT, MacEachern KH, Raby S, et al. Women serving in the Canadian Armed Forces: strengthening military capabilities and operational effectiveness. Can Mil J [Internet]. 2019;19(2):24-33. Available from: http://www.journal.forces.gc.ca/Vol19/No2/ page24-eng.asp.

30. Demers AL. From death to life: female Veterans, identity negotiation, and reintegration into society. J Humanist Psychol. 2013;53(4):489-515. https://doi. org/10.1177/0022167812472395.

31. Messinger SD. Getting past the accident: explosive devices, limb loss, and refashioning a life in a military medical center. Med Anthropol Q. 2010;24(3):281-303. https://doi.org/10.1111/j.15481387.2010.01105.x. Medline:20949837

32. Skomorovsky A, Wan C, Watkins K. Introduction to the financial well-being of Canadian Armed Forces members and families. J Mil Vet Fam Health. 2019;5(S1):1-9. https://doi.org/10.3138/jmvfh.5.s1.2018-0031.

33. MacLean MB, Pound T, Sweet J, et al. Financial security among Veterans in Canada [Internet]. Charlottetown, PE: Research Directorate, Veterans Affairs Canada; 2019.

34. Hachey KK. Transition from military to civilian life: male and female Canadian Armed Forces Veterans. Ottawa, ON: Director General Military Personnel Research and Analysis, Department of National Defence; 2014.

35. Office of the Veterans Ombudsman. Transitioning successfully: a qualitative study. Ottawa, ON: Office of the Veterans Ombudsman; 2017.

36. Daté A, Berlinguette MK. Will my pension be enough? Bridge employment intentions of Canadian Armed Forces members. J Mil Vet Fam Health. 2019;5(S1): 92-100. https://doi.org/10.3138/jmvfh.5.s1.20180030.

37. MacLean MB, Van Til L, Sweet J, et al. Factors associated with work satisfaction among Veterans. J Mil Vet Fam Health. 2018;4(1):33-41. https://doi. org/10.3138/jmvfh.2017-0013.

38. Ahmad F, Jhajj AK, Stewart DE, et al. Single item measures of self-rated mental health: a scoping review. BMC Health Serv Res. 2014;14(1):Article 398. https://doi. org/10.1186/1472-6963-14-398. Medline:25231576

39. Benyamini $Y$. Why does self-rated health predict mortality? An update on current knowledge and a research agenda for psychologists. Psychol Health. 2011;26(11):1407-13. https://doi.org/10.1080/08870 446.2011.621703. Medline:22111660

40. Mawani FN, Gilmour H. Validation of self-rated mental health. Health Rep. 2010;21(3):61-75. 
41. Robinson J, Littlefield P, Schleuning A. Transforming Veterans' experiences during military-to-civilian transition: gaps and opportunities. Washington, DC: US Department of Veterans Affairs Center for Innovation; 2017.

42. Candian Armed Forces Transition Group. My transition guide: transitioning from military to civilian life. Ottawa, ON: Canadian Armed Forces Transition Group, Department of National Defence; 2019.

\section{AUTHOR INFORMATION}

Jennifer E.C. Lee, PhD, is a Defence Scientist at Director General Military Personnel Research and Analysis, Department of National Defence and Chair of The Technical Cooperation Program (TTCP) Human Resources and Performance Group (HUM) Technical Panel 21 on Resilience. Currently, she is Acting Director of Research on Personnel and Family Support and oversees her team's work on a range of topics including: sexual misconduct; diversity and inclusion; personnel and family support programs; and various topics in military, Veteran, and family health.

Sanela Dursun, PhD, is Chief Scientist at Director General Military Personnel Research and Analysis, Department of National Defence (DND). She oversees the centre's program of research, which is aimed at supporting and developing personnel management policies and programs in the Canadian Armed Forces. She has published and disseminated research findings in various domains of personnel research, including military family well-being, diversity, and Veteran's health, both within the DND and in a variety of international fora. She holds a Master's in social psychology and a $\mathrm{PhD}$ in health psychology. Prior to joining DND in 2002, Dr. Dursun worked in the high-tech industry, at Nortel Networks and Corel Corporation.

Alla Skomorovsky, $\mathrm{PhD}$, is a Defence Scientist at Director General Military Personnel Research and Analysis, Department of National Defence, in the Family and Community Support section. Her research interests include quantitative and qualitative research in the areas of health, coping, personality, and well-being of military families. She is also an adjunct professor at Carleton University.

James M. Thompson, MD, was the Research Medical Advisor at Veterans Affairs Canada until March 2019. He served in the CAF Reserve Force prior to medicine and has over 100 scientific publications. He is Adjunct Associate Professor, Department of Public Health Sciences, at Queen's University. His primary research focus is Veterans' wellbeing.

\section{COMPETING INTERESTS}

None declared.

This article has been peer reviewed.

\section{CONTRIBUTORS}

All authors conceived and designed the study. Jennifer E.C. Lee conducted the analyses and edited and revised the manuscript. All authors contributed to drafting the manuscript and approved the final version submitted for publication.

\section{FUNDING}

None declared. 